\title{
Surgery crisis simulation during the COVID-19 pandemic
}

\author{
Alexandra McDonald, BMath \\ Mehdi Qiabi, MD \\ Deb Lewis, BSc \\ Robert Leeper, MD \\ Dalilah Fortin, MD \\ Richard Inculet, MD \\ Richard Malthaner, MD
}

Accepted October 5, 2021

This project's abstract has been presented at the 2020 Canadian Conference for the Advancement of Surgical Education (C-CASE).

\section{Correspondence to: \\ R. Malthaner \\ London Health Sciences Centre \\ 800 Commissioners Rd. E., \\ Suite E2-124 \\ London ON N6A 5W9 \\ Richard.malthaner@Ihsc.on.ca}

Cite as: Can J Surg 2022 March 2; 65(2). doi:10.1503/cjs.025420

\section{SUMmarY}

COVID-19 puts health care providers at risk for infection with SARS-CoV-2 . Personal protective equipment (PPE) can reduce viral transmission if used properly. We used simulation of an intraoperative crisis involving an infectious outbreak to assess PPE adherence and confidence in PPE use. Simulation of an intraoperative crisis with a patient with COVID-19 revealed gaps in PPE adherence; however, simulation training successfully increased confidence in PPE use and received positive feedback.

T he COVID-19 pandemic puts health care workers at risk for infection with SARS-CoV-2 because of direct and prolonged exposure to patients with COVID-19. Personal protective equipment (PPE) can be used to reduce viral transmission. However, proper PPE protocols must be followed in order to successfully reduce viral transmission. Various problems with PPE adherence and ease of use can limit its effectiveness.

Our goal was to explore the use of an in situ intraoperative crisis simulation involving an infectious outbreak as a tool for assessment of PPE adherence, confidence in PPE use, and latent safety threats.

\section{Simulation}

Three simulations were conducted in July 2020 involving 12 participants: 3 senior thoracic surgery trainees, 3 anesthesia residents, and 6 registered nurses. The simulation involved a tracheo-innominate fistula in a mannequin representing a patient with COVID-19; PPE was available. Simulations were video-recorded and reviewed by 2 thoracic surgery consultants to assess adherence to the London Health Sciences Centre (LHSC) Donning and Doffing Sequence for Protected Droplet and Contact With Enhanced PPE (unpublished; Appendix 1, available at www.canjsurg.ca/lookup/doi/10.1503/ cjs.025420/tab-related-content). After simulation, all participants completed forms to assess confidence in PPE use and simulation fidelity. The Simulation PPE Confidence form was based on a 5-point Likert scale, ranging from 1 (not at all confident) to 5 (extremely confident), and asked participants to recall perceived presimulation and actual post-simulation confidence in the following areas: managing a surgical emergency with a COVID-19-positive patient, performing operating room skills while wearing PPE, donning PPE, and doffing PPE. Simulation fidelity was assessed using the Method Material Member Overall (MMMO) Questionnaire, asking participants to rate simulation experience on a scale of 1 (disagree) to 5 (agree)" (Appendix 1). ${ }^{1-3}$

\section{Adherence}

All participants responded "yes" to "Are you aware of the current London Health Sciences Centre (LHSC) guidelines for PPE use?" Video review showed deviations from the LHSC PPE Checklist for all participants during both donning and doffing. Two thoracic surgery consultants 
separately reviewed PPE adherence data, and their assessments were initially 90\% (836/924) in agreement. Any discrepancies were discussed until a final assessment was agreed upon.

Observed use of hand hygiene was $0 \%(0 / 12)$, neck protection was $17 \%(2 / 12)$, inner gloves was $8 \%(1 / 12)$, gown was $100 \%(12 / 12)$, N95 was $83 \%(10 / 12)$, goggles was $0 \%(0 / 12)$, face shield was $92 \%(11 / 12)$, outer bouffant was $75 \%(9 / 12)$, and outer gloves was $92 \%(11 / 12)$ (Figure 1). Participants on average donned $57 \%$ of items in the correct sequence.

Adherence to PPE guidelines was higher for doffing than for donning. Final hand hygiene showed greater than $85 \%$ adherence, and all other doffing checklist items showed $100 \%$ adherence. Participants on average doffed $92 \%$ of items in the correct sequence.

\section{Confidence}

Confidence in performing OR skills while wearing PPE refers to skills including localization of simulated tracheoinnominate fistula, removal of tracheotomy tube, compression of simulated bleeding vessel and intubation with single lumen tube. Surgery trainees were asked to verbally describe how to proceed with further surgical management of a tracheo-innominate fistula. On average, participants increased in confidence in each of the 4 areas assessed after simulation, with an overall confidence increase from 3.9 to 4.2 out of 5 (Figure 2).

On the MMMO Questionnaire (Appendix 1), on average, methods scored 4.6, materials scored 4.6, members scored 4.4, and overall scored 4.8, all out of 5 (Figure 3). Written feedback was also positive.

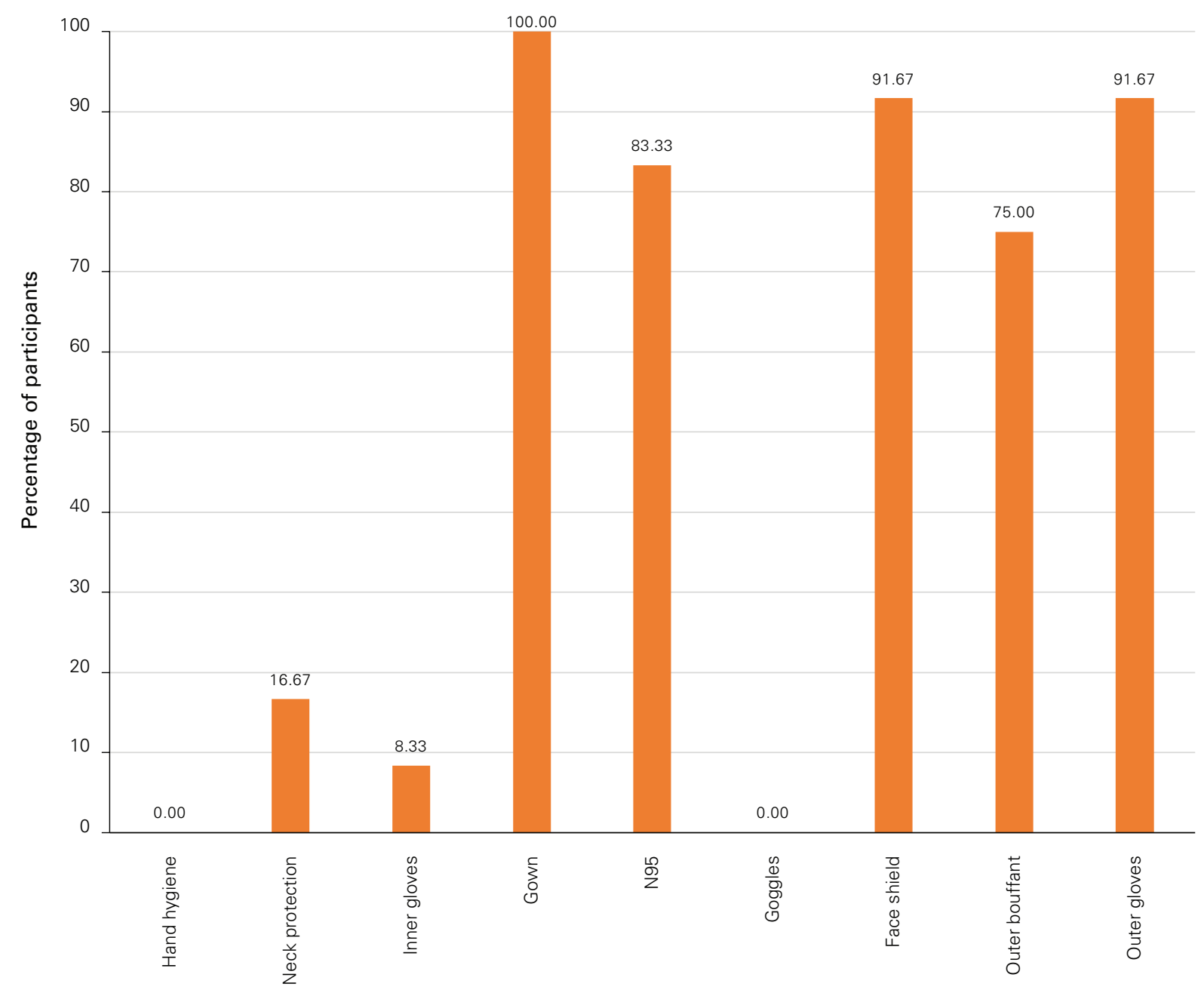

PPE item

Fig. 1. Assessment of PPE donning item adherence, based on the proportion of participants donning items indicated in the London Health Sciences Centre (LHSC) PPE Checklist. PPE = personal protective equipment. 


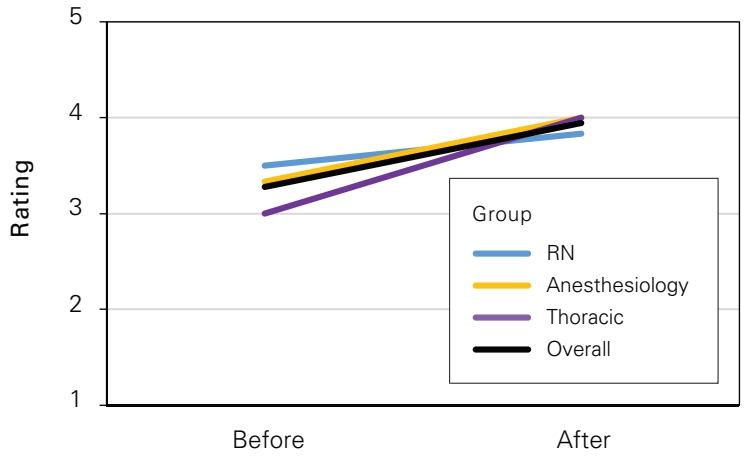

Confidence in managing a surgical emergency with a COVID-19 positive patient

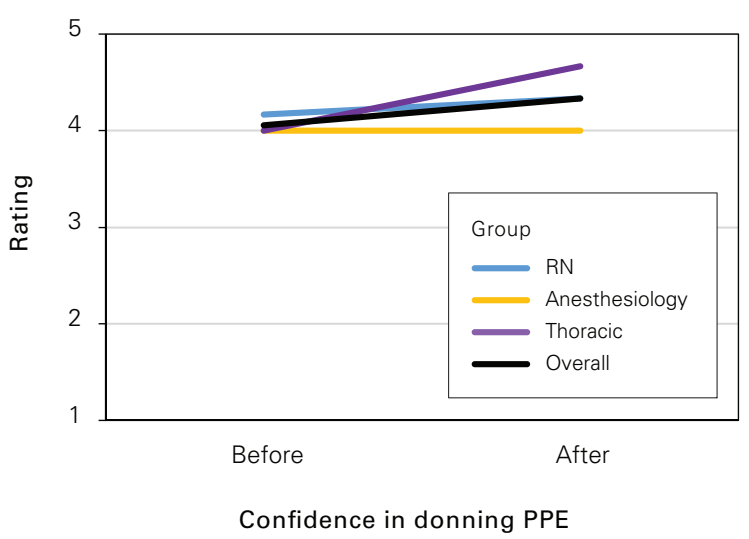

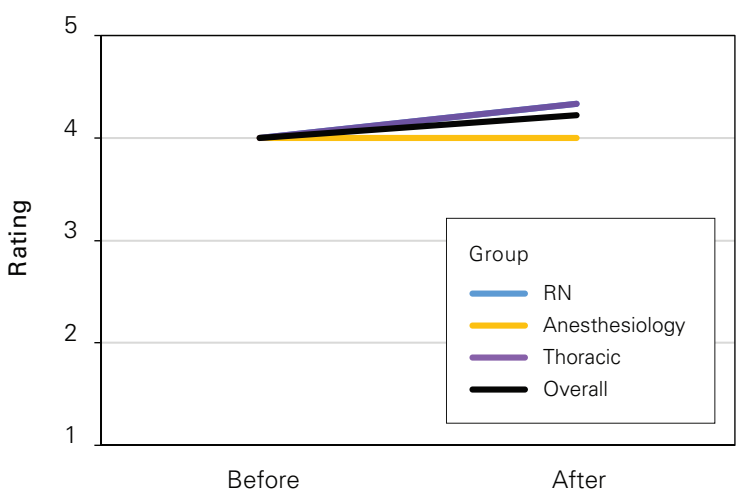

Confidence in performing OR skills while wearing PPE

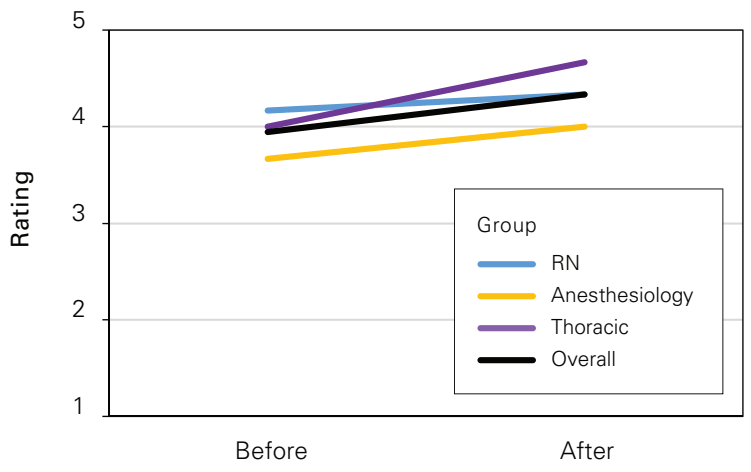

Confidence in doffing PPE

Fig. 2. Scores on the PPE Confidence form before and after simulation and debrief. A total of 3 thoracic surgery trainees, 3 anesthesiology residents and 6 registered nurses completed the form. Recalled presimulation and actual postsimulation confidence were scored in 4 areas: managing a surgical emergency involving a patient with COVID-19, performing OR skills while wearing PPE, donning PPE, and doffing PPE. The scale ranged from 1 (not at all confident) to 5 (extremely confident). Mean scores by role are displayed. $\mathrm{OR}=$ operating room; $\mathrm{PPE}=$ personal protective equipment; $\mathrm{RN}=$ registered nurse.

\section{Discussion}

To our knowledge, we were the first to use an in situ intraoperative crisis simulation involving an infectious outbreak to examine gaps in PPE adherence. Results were below acceptable in both adherence to and sequence of PPE donning and doffing steps. The simulation led to an increase in self-reported confidence surrounding PPE use. Participant feedback confirmed the high fidelity of the simulation model. The results support the need for PPE guideline alterations and encourage the use of simulation to enhance future outbreakrelated preparedness.

Each participant confirmed awareness of the LHSC guidelines for PPE use, yet each participant showed donning and doffing adherence problems. Donning problems included low adherence rates for hand hygiene, neck protection, inner gloves and goggles. The
LHSC guidelines require at minimum hand hygiene and goggles, and further encourage additional precautions with neck protection and inner gloves if these can be used. None of the participants completed pre-donning hand hygiene or donned goggles, meaning that none of the participants met the minimum donning requirements. Furthermore, there was room for improvement in additional precautions with neck protection and inner glove use. Doffing had higher adherence rates. The main problem was below $100 \%$ adherence to the correct sequence of doffing steps. There is a substantial risk of self-contamination when doffing PPE, and problems are related mainly to doffing sequence. ${ }^{4}$ Training health care workers not only on correct doffing steps, but also on correct sequence of doffing steps is critical to mitigate risk of infection.

Multidisciplinary discussions have identified factors contributing to poor PPE adherence. There are 


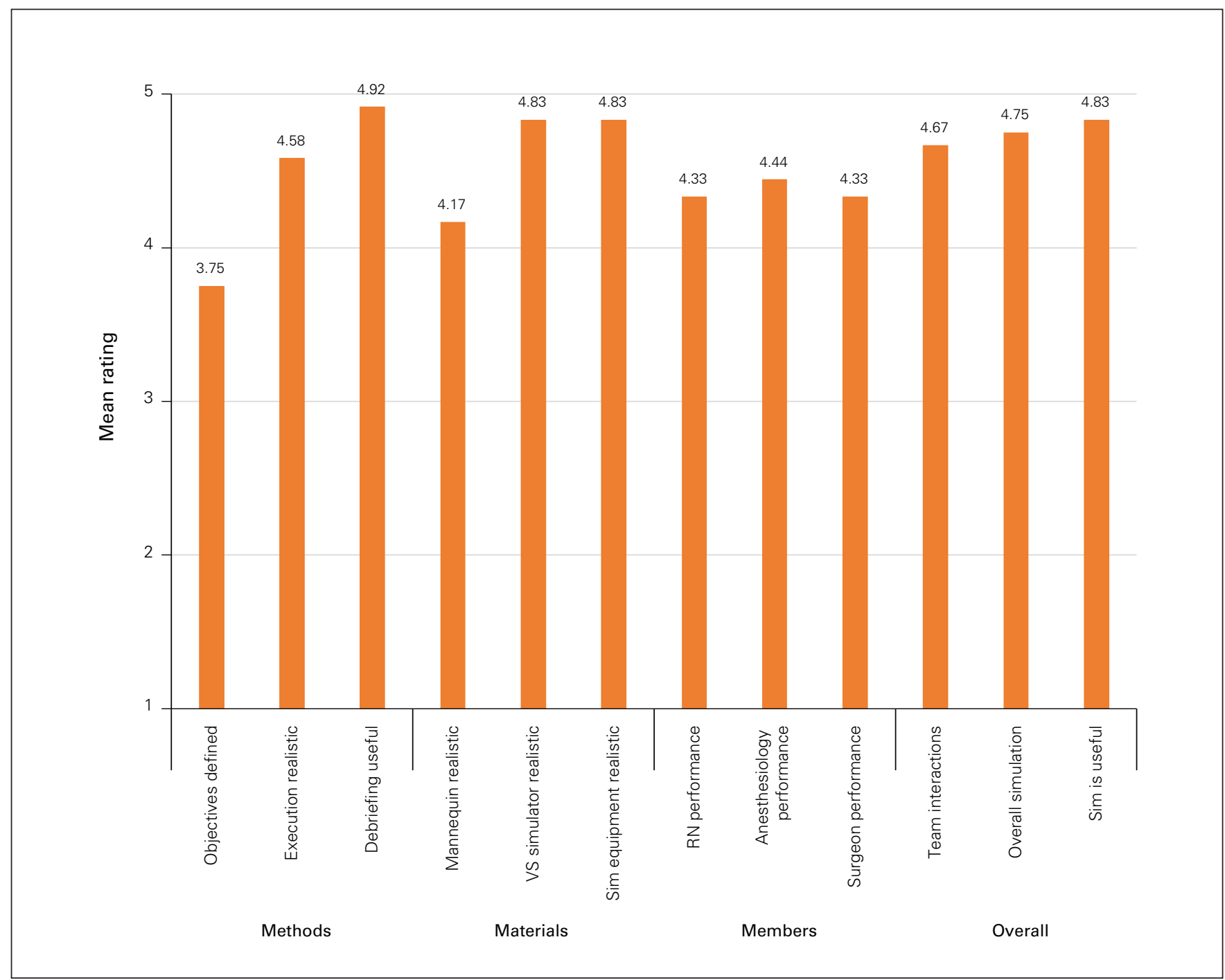

Fig. 3. Material Method Member Overall (MMMO) Questionnaire results. A total of 3 thoracic surgery trainees, 3 anesthesiology residents and 6 registered nurses completed the questionnaire. Simulation experiences were rated on a scale of 1 (disagree) to 5 (agree) and mean scores are displayed. $\mathrm{RN}=$ registered nurse; $\mathrm{Sim}=$ simulation; $\mathrm{VS}=$ vital sign.

discrepancies between the LHSC PPE guidelines and the COVID-19 donning and doffing operating room posters. Notably, neck protection and inner gloves were not indicated on the donning operating room poster. Additionally, extended cuff gloves are required by the LHSC PPE guidelines, but are not supplied by the hospital. Our alternative was to provide medical exam gloves and long sterile gloves, although this change from guidelines may have contributed to participant confusion and lower adherence rates. We recommend updating guidelines to ensure consistency and presenting PPE instructions in a simple visual format. Furthermore, there should be clear indications of what PPE items are required. The current PPE checklist categorizes neck protection and inner gloves as "if used" items, introducing confusion into PPE practice.

Several latent safety threats (LSTs) were identified. One LST stemmed from lack of clear PPE precaution signs. Other LSTs involved equipment failure and pager miscommunications. A final LST was identified regarding extended cuff gloves, as described above.

\section{Conclusion}

Although the number of simulations was small and therefore may not be generalizable, our simulation of an intraoperative crisis involving a patient with COVID-19 revealed gaps in PPE adherence. Simulation training successfully increased confidence levels among participants and received positive feedback with regards to the high fidelity and usefulness of the learning exercise. Furthermore, we were able to identify latent safety threats to improve health care provider and patient safety. We hope that our findings will be useful to LHSC and other institutions to enhance pandemic preparedness and improve safety. 
Affiliations: From the Division of Thoracic Surgery, London Health Science Centre, London, ON (McDonald, Qiabi, Lewis, Inculet, Malthaner); and the Division of General Surgery, London Health Science Centre, London, ON (Leeper).

Competing interests: R. Malthaner is an associate editor of CFS; he was not involved the the review process or decision to accept this paper for publication. No other competing interests were declared.

Contributors: All authors contributed substantially to the conception, writing and revision of this article and approved the final version for publication.

Content licence: This is an Open Access article distributed in accordance with the terms of the Creative Commons Attribution (CC BY-NC-ND 4.0) licence, which permits use, distribution and reproduction in any medium, provided that the original publication is properly cited, the use is noncommercial (i.e., research or educational use), and no modifications or adaptations are made. See: https://creativecommons.org/licenses/by-nc-nd/4.0/

\section{References}

1. O'Keeffe DA, Bradley D, Evans L, et al. Ebola emergency preparedness: simulation training for frontline health care professionals. MedEdPORTAL 2016;12:10433.

2. LoSavio PS, Eggerstedt M, Tajudeen BA, et al. Rapid implementation of COVID-19 tracheostomy simulation training to increase surgeon safety and confidence. Am 7 Otolaryngol 2020;41:102574.

3. Bierer J, Memu E, Leeper WR, et al. Development of an in situ thoracic surgery crisis simulation focused on nontechnical skill training. Ann Thorac Surg 2018;106:287-92.

4. Chughtai AA, Chen X, Macintyre CR. Risk of self-contamination during doffing of personal protective equipment. $A m$ f Infect Control 2018;46:1329-34. 